\title{
Trasplante renal de donante vivo: evaluación de los candidatos mediante TC helicoidal
}

\author{
J.R. Ayuso ${ }^{1}$, F. Openheimer ${ }^{2}$, C. Ayuso ${ }^{1}$, R. Álvarez-Vijande ${ }^{3}$, R. Gutiérrez ${ }^{3}$, A. Lacy ${ }^{4}$, \\ A. Alcaraz ${ }^{3}$, C. Nicolau ${ }^{1}$ \\ ${ }^{1}$ Servicio de Radiodiagnóstico, Centre de Diagnòstic per la Imatge Clínic. ${ }^{2}$ Unidad de Trasplante Renal, Institut \\ de Malalties Nefrourológiques. ${ }^{3}$ Servicio de Urología, Institut de Malalties Nefrourológiques. \\ ${ }^{4}$ Servicio de Cirugía General y Digestiva, Institut de Malalties Digestives. Hospital Clinic, Barcelona.
}

Actas Urol Esp 2006; 30 (2): 145-151

\section{RESUMEN}

TRASPLANTE RENAL DE DONANTE VIVO: EVALUACIÓN DE LOS CANDIDATOS MEDIANTE TC HELICOIDAL

Introducción y objetivos: La tomografia computarizada (TC) ha ganado aceptación en los últimos años en la evaluación vascular de los candidatos a donante vivo de riñón (DVR). Presentamos los hallazgos observados en una serie consecutiva de 102 candidatos y la correlación hallada con los datos quirúrgicos en los individuos que fueron intervenidos.

Métodos: Desde Mayo de1995 hasta Junio de 2005 fueron evaluados mediante TC 102 candidatos a DVR. En 18 de ellos se realizó también angiografia por sustracción digital (ASD). Cincuenta y ocho candidatos fueron finalmente nefrectomizados para la donación. Se han analizado la prevalencia de variantes arteriales y venosas en los estudios TC realizados, así como su correlación con los datos de la ASD y la cirugía.

Resultados: El 44\% de los candidatos presentaron arterias renales supernumerarias en al menos un riñón. El $71 \%$ de riñones derechos y el $76 \%$ de riñones izquierdos tenían pedículo único. La presencia de bifurcaciones precoces fue del $7,8 \%$ y del $15,7 \%$ en cada lado y la de variantes venosas en el $17,5 \%$ y en el $13,7 \%$ en cada lado respectivamente. La sensibilidad de la TC en relación a la ASD para detectar pedículo arterial múltiple fue del $89 \%$, la especificidad del $100 \%$ y la precisión diagnóstica del 97\%. La correlación observada entre la cirugía y la TC en cuanto a la presencia de variantes arteriales fue del 93\%.

Conclusiones: Las variantes de la normalidad en el pedículo vascular renal tienen elevada prevalencia. La TC helicoidal permite obtener toda la información morfológica necesaria para la cirugía con elevada efectividad y en una sola sesión en los candidatos a DVR.

Palabras clave: Tomografia computerizada. Donante. Trasplante renal.

\section{ABSTRACT \\ LIVING DONOR KIDNEY TRANSPLANTATION: HELICAL CT EVALUATION OF CANDIDATES}

Objectives: Computed tomography (CT) is a diagnostic tool that has won acceptance in the past years for the vascular evaluation of living renal donor (LRD) candidates. We show the findings observed in a series of 102 candidates consecutively evaluated in our centre and present the correlation with the surgical data obtained in those individuals finally operated.

Methods: From May 1995 to June 2005, 102 LRD candidates were evaluated with CT. Eighteen were also studied with digital subtraction angiography (DSA). Fifty-eight donors underwent nephrectomy. Prevalence of arterial and venous variants found on CT exams were analyzed, as well as the correlation with surgical and DSA data when they were obtained.

Results: Forty-four percent candidates showed multiple renal arteries at least in one kidney. Seventy-one percent of right kidneys and $76 \%$ of left kidneys had a single arterial pedicle. Early branching was observed in $7.8 \%$ and $15.7 \%$ in right and left kidneys respectively. Venous variants were present in $17.5 \%$ and the $13.7 \%$ in right and left sides respectively. Sensitivity, specificity and accuracy of CT to detect accessory arteries were $89 \%, 100 \%$ and $97 \%$ respectively when it was compared to DSA. Correlation with surgical data raised $93 \%$ for the presence of arterial variants.

Conclussions: Vascular variants in renal pedicle showed a high prevalence. Helical CT can effectively achieve all the information required prior to surgery as a sole imaging procedure in LRD candidates.

Keywords: Computed tomography. Living donor. Kidney transplantation.

Cuadro de abreviaturas: ASD: Angiografía por sustracción digital. DVR: Donante vivo de riñón. MIP: Máxima intensidad de proyección. RM: Resonancia magnética. TC: Tomografía computarizada. TR: Trasplante renal. 
$\mathrm{L}^{2}$ a tasa de obtención de órganos para trasplante en 2004 en España fue de 34,6 por millón de habitantes, la más alta del mundo, y el número de trasplantes renales (TR) realizados en nuestro país en ese año fue de 2.125, según datos de la Organización Nacional de Trasplantes ${ }^{1}$. No obstante, este alto número de TR realizados es insuficiente para cubrir las necesidades de órganos ya que los pacientes incluidos cada año en lista de espera duplican al de la actividad anual de trasplante. Esto ha conducido a la búsqueda de alternativas al trasplante de cadáver que permitan incrementar el número de órganos disponibles. Una de ellas es el TR procedente de un donante vivo ${ }^{2}$. Aunque este tipo de TR representa todavía un pequeño porcentaje de la actividad total de TR en nuestro país (2,9\% en 2004), su número se ha incrementado notablemente en los últimos años. A ello ha contribuido el desarrollo de la cirugía laparoscópica, introducida en $1995^{3}$ que presenta menor morbilidad y un mayor grado de aceptación por parte del donante ${ }^{4,5}$.

La aceptación de un candidato como donante requiere la identificación precisa del número y disposición de estructuras arteriales y venosas del riñón a trasplantar. Los estudios de imagen tienen un papel preeminente en esta evaluación. La angiografia aórtica y selectiva renal y la urografía intravenosa han sido clásicamente las técnicas empleadas en la evaluación de los candidatos a DVR. Sin embargo, en los últimos años la tomografia computarizada y la resonancia magnética (RM) han emergido como nuevos métodos menos agresivos para la evaluación de los candidatos a DVR. En 1995 se inició el estudio de los candidatos a DVR mediante TC helicoidal en nuestro centro.

Se presenta nuestra experiencia en la utilización de la TC helicoidal para la evaluación de los candidatos a DVR en una serie de 102 individuos. Se describe la frecuencia con la que se han hallado variantes de la normalidad en el pedículo arterial y venoso del riñón, así como la correlación de estos datos con los observados en la ASD y la cirugía cuando éstas se llevaron a cabo.

\section{MATERIAL Y MÉTODOS}

Equipos utilizados

Entre Marzo de 1995 y Junio de 2005 se han estudiado mediante TC helicoidal 102 candidatos a DVR. Los estudios se han llevado a cabo en 3 equipos de TC distintos. Catorce candidatos fueron estudiados en un equipo Somatom Plus (Siemens Medical Solutions, Erlangen, Alemania), 16 en un equipo Somatom Plus 4 (Siemens Medical Solutions, Erlangen, Alemania) y 70 en un equipo Somatom Emotion Duo (Siemens Medical Solutions, Erlangen, Alemania). Los dos primeros eran equipos de TC helicoidal monocorte, el primero con limitación del tiempo de disparo del tubo de rayos $\mathrm{x}$ de 24 segundos y el segundo superior a 60 segundos, mientras que el tercero era un equipo bidetector.

\section{Técnica de estudio}

En todos los casos se obtuvieron imágenes no contrastadas sobre el abdomen así como estudio en fase arterial durante la inyeción ev de $140 \mathrm{ml}$ de contraste yodado no iónico (Omnipaque 300; Amersham Health, Oslo, Noruega u Omnitrast 300; Schering, Berlin, Alemania) a través de una vena antecubital, mediante una aguja 20G, a $3 \mathrm{ml} / \mathrm{s}$. En el primer equipo se sincronizó la adquisición del TC helicoidal con la llegada del bolo de contraste a la aorta mediante la administración previa de $10 \mathrm{ml}$ de contraste de prueba, y en los otros dos equipos mediante la utilización de un software específico (Care-Bolus; Siemens, Erlangen, Alemania). El espesor de sección en el primero fue de $3 \mathrm{~mm}$, en el segundo de $2 \mathrm{~mm}$ y en el tercero de $2 \times 1,5$ $\mathrm{mm}$. Se obtuvieron posteriormente imágenes en fase nefrográfica con colimación de $5 \mathrm{~mm}$ sobre áreas renales a los $100 \mathrm{seg}$ del comienzo de la inyección del contraste. Tras finalizar la adquisición de imágenes de TC, los cincuenta últimos candidatos fueron trasladados a una sala de radiología digital situada a pocos metros de distancia, donde se obtuvieron radiografias del abdomen con el fin de obtener el estudio urográfico de la vía excretora renal.

\section{Evaluación de los estudios de TC}

Las imágenes volumétricas obtenidas se trasladaron a una estación de trabajo (3D Virtuoso o 3D Leonardo, Siemens Medical Solutions, Erlangen, Alemania). En ellas se analizaron las imágenes individuales así como reconstrucciones multiplanares o de máxima intensidad de proyección (MIP). Se analizó el número de arterias renales 
presentes en cada lado. Cualquier bifurcación de la arteria renal principal ocurrida a menos de 2 cm. del origen aórtico se catalogó como bifurcación precoz. Se evaluaron también el número y el trayecto de las venas renales. Finalmente se analizaron la presencia de litiasis y masas renales así como la presencia de alteraciones en el resto de estructuras abdominales visualizadas.

\section{Angiografia por sustracción digital}

Dieciocho candidatos a DVR fueron estudiados mediante ASD, en las que se obtuvieron estudios aórticos así como selectivos renales. Los 14 primeros candidatos de la serie, todos ellos evaluados en el primer equipo de TC hasta marzo de 1999, se estudiaron de forma sistemática. Con posterioridad, 4 candidatos adicionales fueron también estudiados mediante este método tras analizar los datos obtenidos en la TC. En estos estudios se evaluaron también el número de arterias renales en cada lado y la presencia de bifurcaciones precoces.

\section{Nefrectomía en el donante}

En 58 candidatos se llevó a cabo la nefrectomía para el trasplante. La elección del riñón a extraer se efectuó en base a los hallazgos de la TC y/o la ASD, escogiéndose el riñón con anatomía más favorable (pedículo único y sin bifurcaciones precoces). Cuando no hubo diferencias entre ambos lados, se escogió el riñón izquierdo por presentar una vena renal más larga. Veinte donantes fueron intervenidos mediante cirugía abierta y 38 mediante cirugía laparoscópica, estos últimos desde marzo de 2002, fecha en la que se introdujo esta técnica en nuestro centro. Siete candidatos incluidos en esta serie están completando su evaluación. Los motivos por los que no se llevó a cabo la donación en los restantes 37 candidatos fueron: la obtención de un riñón procedente de cadáver en el transcurso del proceso de evaluación (9), alteraciones en pruebas bioquímicas del riñón (9) o generales (7), tamaño renal inadecuado para receptores pediátricos (4), anomalias vasculares bilaterales (2), negativa a la donación (2), alteraciones urográficas (2) y litiasis renal (1). En una candidata no se realizó finalmente la nefrectomía, aunque la cirugía laparoscópica ya había comenzado, por no hallarse un lugar adecuado para la anastomosis vascular en el receptor.

\section{Análisis estadístico}

Se ha descrito la frecuencia de aparición de variantes vasculares arteriales y venosas renales en los individuos de la presente serie. Se ha utilizado la prueba exacta de Fisher para evaluar si existían asociaciones con significación estadística entre la presencia de un pedículo arterial múltiple y de bifurcaciones precoces, por un lado, y de anomalias venosas por otro. El valor de significación se estableció como $\mathrm{p}<0,05$. Se estudió la correlación hallada entre la TC y la cirugía en cuanto al número de arterias renales, la presencia de bifurcaciones precoces y de anomalias venosas. Se calculó la sensibilidad, especificidad y precisión diagnóstica en la determinación del número de arterias renales en los 18 candidatos en los que se realizó ASD.

\section{RESULTADOS}

Cincuenta y siete candidatos (55,9\%) eran portadores de un pedículo arterial único en ambos riñones y otros 37 (36,2\%) presentaban un pedículo simple en un lado (Fig. 1). El pedículo múltiple bilateral sólo fue observado en 8 ocasiones $(7,8 \%)$. En cuanto a la distribución del número de arterias renales en cada lado, hubo pocas diferencias entre ambos. En 73 ocasiones fue observado un pedículo único en el lado derecho (71\%) y en 78 en el izquierdo (76\%); en 26 (25\%) y $19(18,6 \%)$ ocasiones se hallaron dos

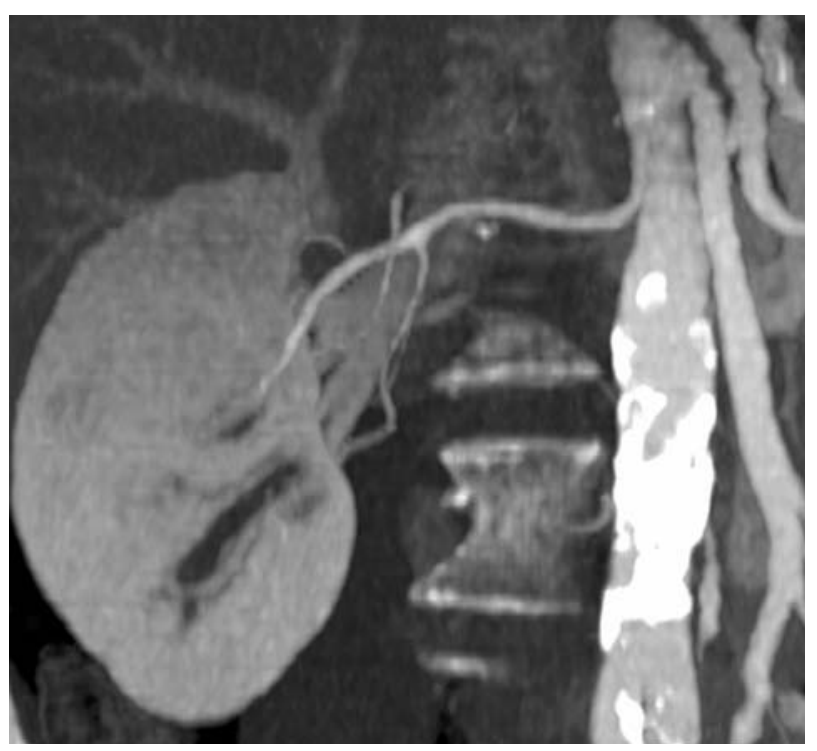

FIGURA 1. Proyección coronal oblicua que muestra una arteria renal derecha única. 
arterias en los lados derecho e izquierdo respectivamente y en $3(3 \%)$ y en $5(5 \%)$ ocasiones se hallaron tres o más arterias en los lados derecho e izquierdo respectivamente. Ocho riñones derechos $(7,8 \%)$ y 16 izquierdos (15,7\%) fueron diagnosticados de bifurcaciones precoces (Fig. 2). No hubo asociación estadísticamente significativa entre la presencia de múltiples arterias renales y de bifurcaciones precoces en el lado derecho $(p=0,68)$ ni en el lado izquierdo $(p=0,34)$ al aplicar la prueba exacta de Fisher.

Las anomalías venosas estuvieron presentes en 21 riñones $(17,5 \%)$ en el lado derecho y en 14 riñones $(13,7 \%)$ en el lado izquierdo. En el lado derecho predominaron las venas de drenaje múltiples ( 2 venas en 20 riñones y 3 en 1 riñón). En el lado izquierdo el pedículo venoso múltiple sólo se apreció en 2 individuos. Las anomalías más frecuentes se observaron en la disposición de las venas: 7 mostraban una distribución circumaórtica (Fig. 3) y 5 retroaórtica. No hubo asociación estadísticamente significativa entre la presencia de múltiples arterias renales y de anomalías venosas en el lado derecho $(\mathrm{p}=0,34) \mathrm{ni}$ en el lado izquierdo $(\mathrm{p}=0,72)$ al aplicar la prueba exacta de Fisher.

En 18 candidatos se dispuso de estudio angiográfico para comparar con la TC. Veintisiete riñones tenían una arteria en la ASD, 7 tenían 2 arterias y 2 tenían 3 arterias. Hubo concordancia en cuanto al número de arterias observadas en 35 de los 36 riñones evaluados (Fig. 4). En una ocasión la ASD indicó la presencia de una arteria supernumeraria de forma indirecta al observar

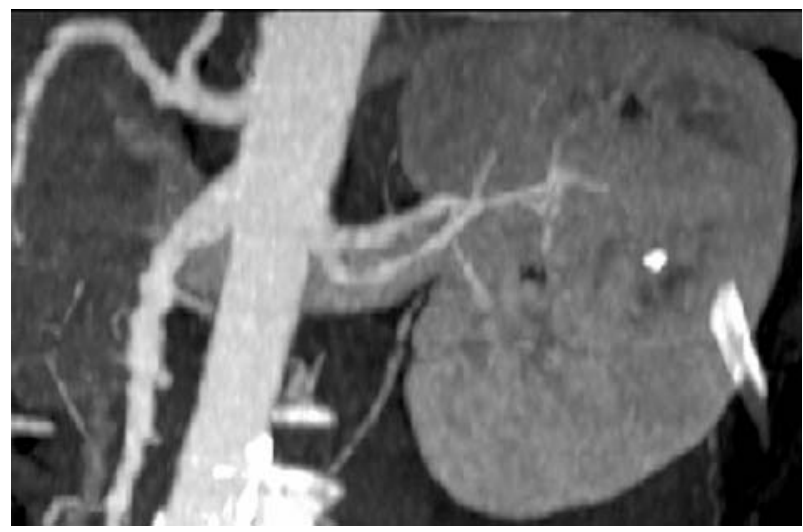

FIGURA 2. Bifurcación de la arteria renal izquierda que ocurre a menos de $1 \mathrm{~cm}$ de su origen aórtico. Se aprecia también una pequeña litiasis renal.

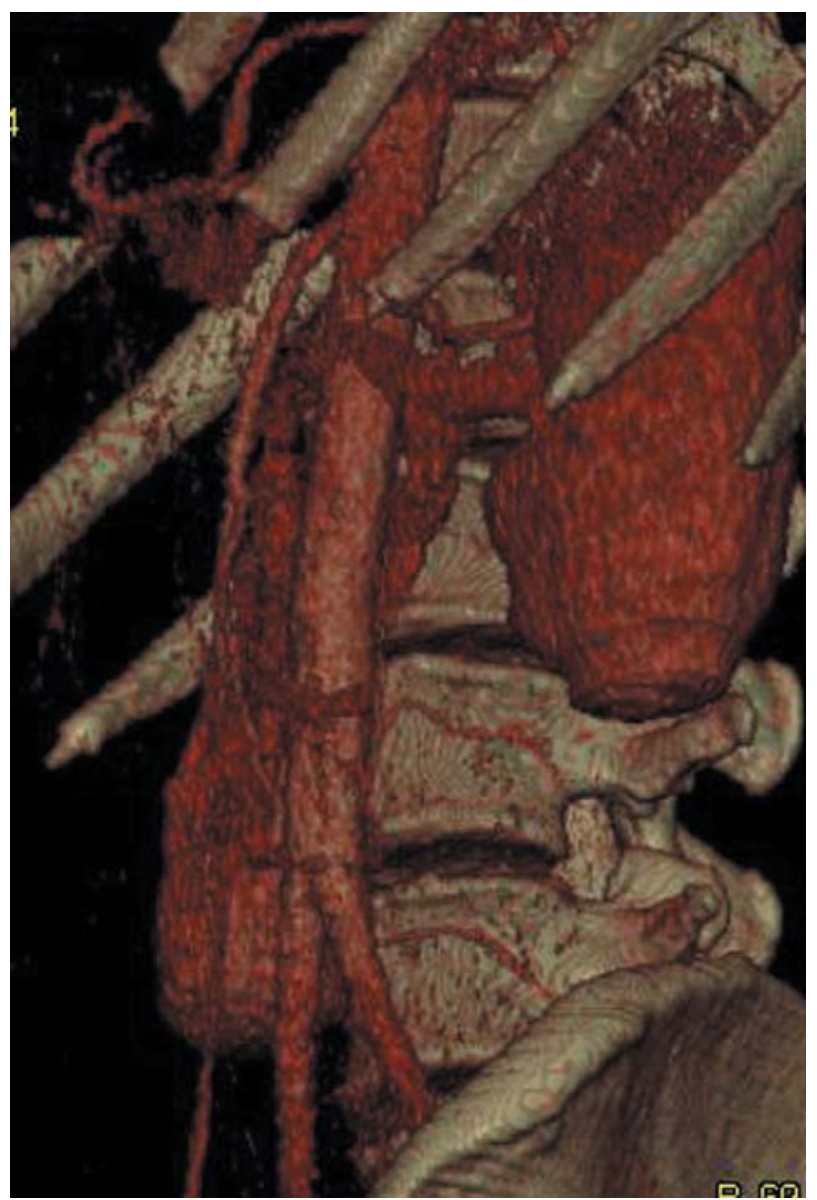

FIGURA 3. Proyección oblicua izquierda que muestra una vena renal izquierda en el hilio renal que se separa en dos venas, una con trayecto preaórtico y otro retroaórtico antes de entrar en la vena cava inferior.

un pequeño defecto en el nefrograma. Sin embargo su extensión era mínima, indicando que el tamaño de la arteria que irrigaba ese fragmento era pequeña. Por ello no se procedió a la búsqueda intencionada y selectiva de la misma. La sensibilidad para detectar pedículo arterial múltiple fue del $89 \%$, la especificidad del $100 \%$ y la precisión diagnóstica del 97\%.

En 58 ocasiones los hallazgos de la TC se correlacionaron con los de la cirugía. Éstos fueron coincidentes en cuanto al número de arterias en 54 ocasiones (93\%), mostrando 53 riñones una sola arteria y 1 riñón dos arterias. Se trataba de una arteria polar muy pequeña que se seccionó y se ligó en el acto quirúrgico y que no se anastomosó en el receptor. En 4 ocasiones se hallaron pedículos múltiples en la cirugía que no habían sido reconocidos previamente en la TC. 


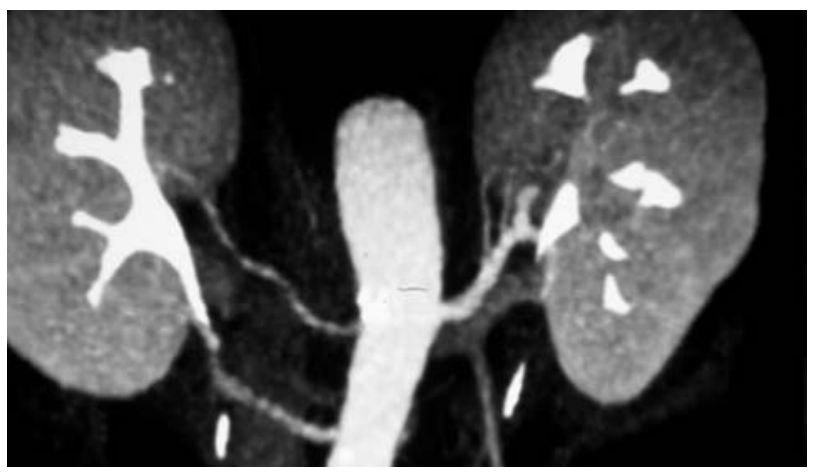

FIGURA 4a. Proyección MIP (arriba) que muestra dos arterias renales dominantes en el lado derecho y sección axial de TC (abajo) en la que se aprecia una tercera arteria de situación craneal.

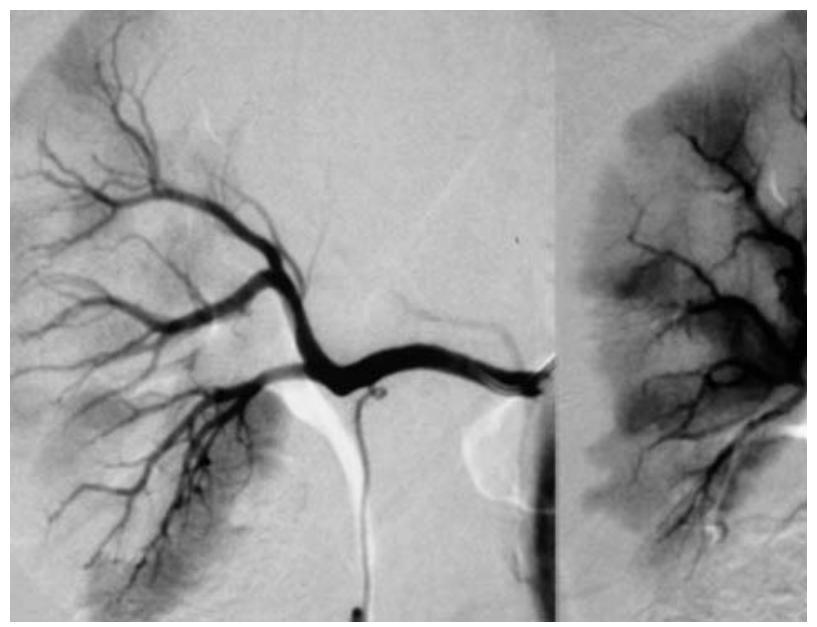

FIGURA 4b. Este hallazgo es coincidente con el observado en la angiografia selectiva.

En 2 ocasiones se trataba de 2 arterias renales adyacentes, que fueron identificadas como independientes de forma retrospectiva (Fig. 5). En otro individuo se ligó una pequeña arteria polar, inferior a 2 $\mathrm{mm}$. que no pudo reconocerse al reevaluar de nuevo el examen de TC. En el último caso, se hallaron 3 arterias en la cirugía. Sólo 2 de ellas se vieron de forma retrospectiva, en un estudio muy degradado por marcados movimientos respiratorios. En ningún caso se produjo desestimación del riñón en el quirófano o pérdida del injerto obtenido atribuible a la falta de reconocimiento de variantes arteriales.

Cinco de los riñones extirpados eran portadores de bifurcaciones precoces y en los cinco se reconoció la anomalía observada en la TC. En todos los casos se realizó una única anastomosis arterial en el receptor. Sólo en un caso se ligó una

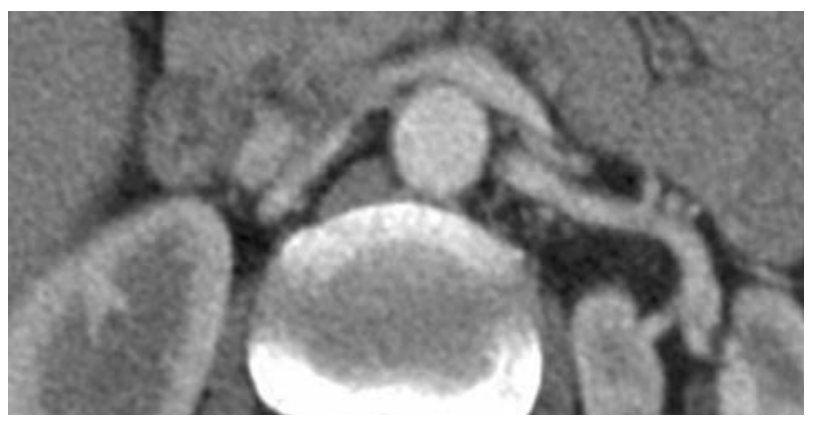

FIGURA 5. Imagen axial de TC en la que se aprecia una arteria renal izquierda dominante y una arteria renal accesoria situada entre la arteria principal y la vena renal. Esta arteria sólo fue reconocida de forma retrospectiva tras la nefrectomia.

pequeña arteria inferior a $2 \mathrm{~mm}$ que se originaba a menos de $\mathrm{cm}$ del ostium y que penetraba a través del córtex renal suprahiliar.

En cuanto a la correlación quirúrgica de las variantes venosas, solo estuvieron presentes en 6 nefrectomías izquierdas y consistieron en 4 venas de localización retroaórtica, 1 circumaórtica y un riñón con 2 venas renales. Todas ellas habían sido detectadas en la TC y fueron confirmadas en la cirugia. En las restantes nefrectomías practicadas no se describieron variantes en el número ni disposición de la vena renal que discreparan de los hallazgos previamente observados en la TC.

\section{DISCUSIÓN}

El estudio radiológico es una parte crucial del proceso de evaluación del candidato a DVR. El análisis detallado de la anatomía vascular arterial y venosa renal y del sistema colector permite seleccionar el riñón técnicamente más sencillo para la nefrectomía. Aunque la presencia de múltiples arterias en el injerto no afecta de forma adversa a la supervivencia del injerto o del paciente, su manejo quirúrgico es más complejo ${ }^{6}$. Los estudios de imagen también permiten detectar patología renal o abdominal que pueden alterar la decisión de la donación. Clásicamente esta información se había obtenido mediante la ASD y la urografia intravenosa. La ASD está considerada como el patrón de oro en la evaluación de la anatomía arterial renal ${ }^{7}$. Sin embargo, en los últimos años ha sido progresivamente sustituida por la TC helicoidal y en algunos centros por la RM, que han demostrado su eficiencia con menor agresividad ${ }^{8-10}$. 
La presencia de variantes en el número y distribución de las arterias y venas renales tiene una alta prevalencia en la población normal y se detecta mediante técnicas de imagen en 44-48\% de los individuos ${ }^{11,12}$. Esta prevalencia es similar a la observada en los individuos de nuestra serie y se da con frecuencia similar en ambos lados. La presencia de más de una arteria en un riñón no se ha asociado de forma significativa a otras variantes como bifurcaciones precoces o anomalías venosas en los individuos evaluados.

Durante los primeros años en los que los candidatos a DVR se evaluaron mediante TC en nuestro centro se constató una elevada concordancia con la información aportada por la ASD, por lo que ya en 1999 se decidió utilizar la TC helicoidal como técnica de estudio inicial. La correlación observada en la literatura entre la TC y los hallazgos quirúrgicos oscila entre el 90\% y el $97 \%$ en series con gran número de donantes ${ }^{10,13-15}$. Estos resultados son similares a los observados en series más recientes tras la introducción de equipos de TC con múltiples detectores $^{16,17}$. La correcta correlación con los hallazgos quirúrgicos es mayor para riñones con pedículos arteriales únicos y disminuye para pedículos arteriales múltiples ${ }^{13}$. Los resultados observados en nuestra serie están en línea con los publicados en la literatura. La mayor tasa de correlación quirúrgica observada en riñones con una sola arteria está influenciada por la selección prequirúrgica para la donación de riñones con aporte arterial simple en las pruebas de imagen. Sólo en una ocasión en nuestros donantes se escogió para la cirugía un riñón con dos arterias detectadas previamente en la TC.

Las arterias que con mayor frecuencia pasan por alto en la TC tienen pequeño tamaño, generalmente menos de $2 \mathrm{~mm}$. Aunque se sacrifiquen en la cirugía, no interfieren con la evolución del injerto ya que la pérdida de volumen renal que suponen es despreciable ${ }^{14}$. Además de su tamaño, otras causas que han conducido a infraestimar el número de arterias en nuestra serie han sido la proximidad en el trayecto de las dos arterias y la obtención de un estudio subóptimo por artefactos de respiración. El hecho de que, en el primer caso, la correcta anatomía arterial fuera detectada de forma retrospectiva enfatiza la importancia de un análisis cuidadoso de las imágenes en la estación de trabajo. En el segundo caso, debe repetirse el examen a fin de obtener la información adecuada para la cirugía. La presencia de bifurcaciones precoces, en los casos intervenidos, no presentó problemas quirúrgicos ya que tenían lugar a más de $1 \mathrm{~cm}$ del ostium aórtico de la arteria renal. La correlación de esta variante con los hallazgos quirúrgicos fue, por lo tanto, excelente.

La correlación observada entre la TC y la cirugía en cuanto a las variantes venosas fue también excelente. Debe destacarse que no se han incluido como variantes venosas las bifurcaciones precoces ni los pedículos venosos complejos, generalmente en relación con venas de drenaje lumbar o gonadal en la vecindad de la vena renal izquierda. Antes de la cirugía laparoscópica estas alteraciones presentaban menos problemática quirúrgica, por lo que no se reflejaban sistemáticamente en los estudios de imagen y por lo tanto no se evaluaron en un número significativo de los estudios de la serie. Aunque su presencia no condiciona la elección del riñón a resecar, la reducción en el campo de visión asociada a esta técnica aumenta la necesidad de conocer la situación de la vena renal y a sus tributarias principales antes de la cirugia ${ }^{14,18}$. El realce observado en el sistema venoso renal en los estudios de TC helicoidal que hemos obtenido en nuestros equipos ha sido en general excelente. Sin embargo, ha sido descrito un escaso realce venoso de hasta un 7\% en la vena adrenal y de un 16\% en la vena gonadal que en ocasiones puede interferir con su identificación, en adquisiciones con TC multidetector en fase arterial tardia ${ }^{19,20}$.

La sustitución de la urografía clásica en los candidatos a DVR por la obtención de imágenes radiológicas del abdomen tras finalizar el estudio $\mathrm{TC}^{21}$ no ha comportado merma alguna en la calidad de la visualización de la vía excretora renal y es una práctica muy aceptada por parte del equipo de trasplante en nuestro centro. El tiempo que transcurre entre el inicio de la inyección del contraste y la obtención de estas imágenes es de aproximadamente 6 a $8 \mathrm{~min}$. Con equipos de TC multidetector es posible obtener también imágenes urográficas en la propia unidad de TC y tienen el potencial de sustituir a la radiología convencional en el estudio de la vía urinaria ${ }^{22}$. 
La angiografia por RM ha sido empleada con éxito por otros autores en el estudio de los candidatos a DVR ${ }^{23}$. La menor disponibilidad de equipos, la menor resolución espacial que se consigue con ésta técnica (aunque esto último puede quedar minimizado en equipos de RM e última generación) y la imposibilidad de identificar calcificaciones favorecen el empleo de la TC. La utilización de radiaciones ionizantes así como el empleo de contrastes yodados gravan no obstante a esta última.

\section{CONCLUSIONES}

El TR de donante vivo es una alternativa al TR de cadáver. Las variantes de la normalidad en el pedículo vascular renal tienen elevada prevalencia. La TC helicoidal, combinada con la obtención de imágenes urográficas morfológicas al concluir el estudio, permite obtener en una sola sesión toda la información necesaria para la nefrectomía laparoscópica con una elevada efectividad.

\section{REFERENCIAS}

1. Organización Nacional de Trasplantes. http://www.msc.es/ Diseno/informacionProfesional/profesional_trasplantes.htm. Accecido en Julio de 2005. 2005.

2. Plaza JJ. Living donor renal transplantation. Nefrologia 2001;21 Suppl 4:97-100.

3. Ratner LE, Ciseck LJ, Moore RG, Cigarroa FG, Kaufman HS, Kavoussi LR. Laparoscopic live donor nephrectomy. Transplantation 1995;60:1047-1049.

4. Kuo PC, Johnson LB, Sitzmann JV. Laparoscopic donor nephrectomy with a 23-hour stay: a new standard for transplantation surgery. Ann Surg 2000;231:772-779.

5. Alcaraz A, Rosales A, Palou J, Caffaratti J, Montlleo M, Segarra J, et. al. Living donor nephrectomy for kidney transplantation. Experience in the first two years. Arch Esp Urol 2004;57:10911098.

6. Ali-El-Dein B, Osman Y, Shokeir AA, Shehab El-Dein AB, Sheashaa $\mathrm{H}$, et. al. Multiple arteries in live donor renal transplantation: surgical aspects and outcomes. J Urol 2003;169: 2013-2017.

7. Spencer W, Streem SB, Geisinger MA, Novick AC, Steinmuller DR, Zelch MG, et. al. Outpatient angiographic evaluation of living renal donors. J Urol 1988;140:1364-1366.

8. Low RN, Martinez AG, Steinberg SM, Alzate GD, Kortman KE, Bower BB, et. al. Potential renal transplant donors: evaluation with gadolinium-enhanced MR angiography and MR urography. Radiology 1998;207:165-172.

9. Cochran ST, Krasny RM, Danovitch GM, Rajfer J, Barbaric ZM, Wilkinson A, et. al. Helical CT angiography for examination of living renal donors. AJR Am J Roentgenol 1997;168:1569-1573.

10. Platt JF, Ellis JH, Korobkin M, Reige K. Helical CT evaluation of potential kidney donors: findings in 154 subjects. AJR Am J Roentgenol 1997;169:1325-1330.

11. Spring DB, Salvatierra O, Jr., Palubinskas AJ, Amend WJ, Jr., Vincenti FG, Feduska NJ. Results and significance of angiography in potential kidney donors. Radiology 1979;133:45-47.
12. Cochran ST, Krasny RM, Danovitch GM, Rajfer J, Barbaric ZM, Wilkinson A, et. al. Helical CT angiography for examination of living renal donors. AJR Am J Roentgenol 1997; 168:1569-1573.

13. Janoff DM, Davol P, Hazzard J, Lemmers MJ, Paduch DA, Barry JM. Computerized tomography with 3-dimensional reconstruction for the evaluation of renal size and arterial anatomy in the living kidney donor. J Urol 2004;171:27-30.

14. Del Pizzo JJ, Sklar GN, You-Cheong JW, Levin B, Krebs T, Jacobs SC. Helical computerized tomography arteriography for evaluation of live renal donors undergoing laparoscopic nephrectomy. J Urol 1999;162:31-34.

15. Platt JF, Ellis JH, Korobkin M, Reige K. Helical CT evaluation of potential kidney donors: findings in 154 subjects. AJR Am J Roentgenol 1997;169:1325-1330.

16. Patil UD, Ragavan A, Nadaraj, Murthy K, Shankar R, Bastani B, et. al. Helical CT angiography in evaluation of live kidney donors. Nephrol Dial Transplant 2001;16:1900-1904.

17. Sahani DV, Rastogi N, Greenfield AC, Kalva SP, Ko D, Saini S, et. al. Multi-detector row CT in evaluation of 94 living renal donors by readers with varied experience. Radiology 2005;235:905-910.

18. Kawamoto S, Montgomery RA, Lawler LP, Horton KM, Fishman EK. Multidetector CT angiography for preoperative evaluation of living laparoscopic kidney donors. AJR Am J Roentgenol 2003; 180:1633-1638.

19. Janoff DM, Davol P, Hazzard J, Lemmers MJ, Paduch DA, Barry JM. Computerized tomography with 3-dimensional reconstruction for the evaluation of renal size and arterial anatomy in the living kidney donor. J Urol 2004;171:27-30.

20. Del Pizzo JJ, Sklar GN, You-Cheong JW, Levin B, Krebs T, Jacobs SC. Helical computerized tomography arteriography for evaluation of live renal donors undergoing laparoscopic nephrectomy. J Urol 1999;162:31-34.

21. Lewis GR, Mulcahy K, Brook NR, Veitch PS, Nicholson ML. A prospective study of the predictive power of spiral computed tomographic angiography for defining renal vascular anatomy before live-donor nephrectomy. BJU Int 2004;94:1077-1081.

22. Del Pizzo JJ, Sklar GN, You-Cheong JW, Levin B, Krebs T, Jacobs SC. Helical computerized tomography arteriography for evaluation of live renal donors undergoing laparoscopic nephrectomy. J Urol 1999;162:31-34.

23. Kawamoto S, Lawler LP, Fishman EK. Evaluation of the renal venous system on late arterial and venous phase images with MDCT angiography in potential living laparoscopic renal donors. AJR Am J Roentgenol 2005; 184:539-545.

24. Del Pizzo JJ, Sklar GN, You-Cheong JW, Levin B, Krebs T, Jacobs SC. Helical computerized tomography arteriography for evaluation of live renal donors undergoing laparoscopic nephrectomy. J Urol 1999;162:31-34.

25. Platt JF, Ellis JH, Korobkin M, Reige KA, Konnak JW, Leichtman AB. Potential renal donors: comparison of conventional imaging with helical CT. Radiology 1996;198:419-423.

26. Noroozian M, Cohan RH, Caoili EM, Cowan NC, Ellis JH. Multislice CT urography: state of the art. Br J Radiol 2004;77 Spec No 1:S74-S86.

27. Rankin SC, Jan W, Koffman CG. Noninvasive imaging of living related kidney donors: evaluation with CT angiography and gadolinium-enhanced MR angiography. AJR Am J Roentgenol $2001 ; 177: 349-355$

Dr. J.R. Ayuso

Servicio de Radiodiagnóstico.

Centre de Diagnòstic per la Imatge Clínic

Hospital Clinic

C/ Villarroel 17008036 Barcelona

E-mail: jrayuso@clinic.ub.es

(Trabajo recibido el 13 de septiembre 2005) 